\title{
Effects of Oral Health Related To General Health
}

\author{
R. Sahitha
}

\begin{abstract}
Oral infections especially periodontitis, as a causal factor for systemic diseases. Three mechanisms or pathways linking oral infections to secondary systemic effects have been proposed: (i) metastatic spread of infection from the oral cavity as a result of transient bacteremia, (ii) metastatic injury from the effects of circulating oral microbial toxins, and (iii) metastatic inflammation caused by immunological injury induced by oral microorganisms. These infections are predominantly anaerobic, with gram-negative rods being the most common isolates. The anatomic closeness of this microflora to the bloodstream can facilitate bacteremia and systemic spread of bacterial by-products and immunocomplexes. A variety of clinical procedures such as tooth extraction, periodontal and endodontic treatment, may cause translocation of microorganisms from the oral cavity to the bloodstream. The microorganisms that gain entrance to the blood circulate throughout the body, and causes systemic diseases like cardiovascular disease, bacterial pneumonia, diabetes mellitus, and low birth weight.
\end{abstract}

\section{Introduction}

Focal infection is a localized or generalized infection caused by the dissemination of microorganisms or toxic products from a focus of infection. Focus of infection refers to a circumscribed area of tissue, which is infected with exogenous pathogenic micro organisms and is usually located near a mucous or cutaneous surface. About a century ago, William Hunter synthesized that oral microorganisms and their products were involved in a range of systemic disease. ${ }^{2}$ Oral foci of infection have been related to general health. Oral cavity can act as the site of origin for dissemination of pathogenic organisms to distant body sites, especially in immunocompromised hosts such as patients suffering from malignancies, diabetes, or rheumatoid arthritis or having corticosteroid or other immunosuppressive treatment. The source of infection could be infected periapical lesions such as periapical granuloma, cysts .abscess or teeth with infected root canals or periodontal disease.

\section{Foci Of Infection}

Periodontal disease is caused by bacteria found in dental plaque, and about 10 species have been identified as putative pathogens in periodontal disease, mainly gram-negative rods. ${ }^{2}$ Actinobacillus actinomycetemcomitans, Porphyromonas gingivalis, and Bacteroides forsythus are the gram-negative bacteria most commonly associated with periodontitis . ${ }^{1,3}$ The incidence of bacteremia following dental procedures such as tooth extraction, endodontic treatment, periodontal surgery, and root scaling has been well documented dissemination of oral microorganisms into the bloodstream is common, and less than 1 min after an oral procedure, organisms from the infected site may have reached the heart, lungs, and peripheral Blood capillary system ${ }^{4}$ In the oral cavity there are several barriers to bacterial penetration from dental plaque into the tissue: a physical barrier composed of the surface epithelium; defensins, which are host-derived peptide antibiotics, in the oral mucosal epithelium; an electrical barrier that reflects the Eh difference between the host cell and the microbial layer; an immunological barrier of antibody-forming cells; and the reticuloendothelial system (phagocyte barrier) . ${ }^{5,6}$ Under normal circumstances, these barrier systems work together to inhibit and eliminate penetrating bacteria. When this state of equilibrium is disturbed by an overt breach in the physical system (e.g., trauma), the electrical system (i.e., hypoxia), or immunological barriers (e.g., through neutropenia, AIDS, or immunosuppressant therapy), organisms can propagate and cause both acute and chronic infections with increased frequency and severity . ${ }^{7}$ With normal oral health and dental care, only small numbers of mostly facultative bacterial species gain access to the bloodstream. However, with poor oral hygiene, the numbers of bacteria colonizing the teeth, especially supragingivally, could increase 2 - to 10 -fold ${ }^{8}$ and thus possibly introduce more bacteria into tissue and the bloodstream, leading to an increase in the prevalence and magnitude of bacteremia. The purpose of this review is to show that oral disease especially periodontal disease can be the causative factor for systemic disease.

\section{Pathways Linking Oral Infection To Nonoral Disease:}

Three mechanisms have been proposed for linking oral infections to systemic disease. They are (i) metastatic spread of infection from the oral cavity as a result of transient bacteremia,(ii) metastatic injury from the effects of circulating oral microbial toxins, (iii) metastatic inflammation caused by immunological injury induced by 
oral microorganisms. ${ }^{9}$ METASTATIC INFECTION: oral infections and dental procedures can cause transient bacteremia. The microorganisms that gain entrance to the blood and circulate throughout the body are usually eliminated by the reticuloendothelial system within minutes (transient bacteremia) and as a rule lead to no other clinical symptoms than possibly a slight increase in body temperature . ${ }^{10,11}$ However, if the disseminated microorganisms find favorable conditions, they may settle at a given site and, after a certain time lag, start to multiply.

Metastatic injury: Some gram-positive and gram-negative bacteria have the ability to produce diffusible proteins, or exotoxins, which include cytolytic enzymes and dimeric toxins with A and B subunits. The exotoxins have specific pharmacological actions and are considered the most powerful and lethal poisons known. Conversely, endotoxins are released after cell death .Endotoxin is compositionally a lipopolysaccharide (LPS) that, when introduced into the host, gives rise to a large number of pathological manifestations. LPS is continuously shed from periodontal gram-negative rods during their growth in vivo.$^{12,13}$

Metastatic inflammation: Soluble antigen may enter the bloodstream, react with circulating specific antibody, and form a macromolecular complex. These immunocomplexes may give rise to a variety of acute and chronic inflammatory reactions at the sites of deposition.$^{14}$

\section{Cardiovascular Disease:}

\section{Systemic Diseases Associated With Oral Infection}

Cardiovascular diseases such as atherosclerosis, myocardial Infarction and can occur as a result of a complex set of genetic and environmental factors .The genetic factors include age, lipid metabolism, obesity, hypertension, diabetes, increased fibrinogen levels, and platelet-specific antigen Zwb (P1A2) polymorphism. Environmental risk factors include socioeconomic status, exercise stress, diet, nonsteroidal anti-inflammatory drugs, smoking, and chronic infection. The classical risk factors of cardiovascular disease such as hypertension, hypercholesterolemia, and cigarette smoking can only account for one-half to two-thirds of the variation in the incidence of cardiovascular disease .oral bacteria such as Streptococcus sanguis and Porphyromonas gingivalis induce platelet aggregation, which leads to thrombus formation.

Periodontal disease may stimulate the liver to produce C-reactive protein (CRP) (a marker of inflammation), which in turn will form deposits on injured blood vessels. CRP binds to cells that are damaged and fixes complement, which activates phagocytes, including neutrophils. These cells release nitric oxide, thereby contributing to atheroma formation. ${ }^{15}$

\section{Atherosclerosis}

Atherosclerosis has been defined as a progressive disease process that involves the large- to mediumsized muscular and large elastic arteries. The advanced lesion is the atheroma, which consists of elevated focal intimal plaques with a necrotic central core containing lysed cells, cholesterol ester crystals, lipid-laden foam cells, and surface plasma proteins, including fibrin and fibrinogen ${ }^{16}$ The presence of atheroma tends to make the patient thrombosis prone because the associated surface enhances platelet aggregation and thrombus formation that can occlude the artery or be released to cause thrombosis, coronary heart disease, and stroke.

\section{Myocardial Infarction}

A myocardial infarction is the damaging or death of an area of the heart muscle resulting from a reduced blood supply to that area. Myocardial infarction is almost always due to the formation of an occlusive thrombus at the site of rupture of an

atheromatous plaque in a coronary artery.$^{15}$

\section{Stroke}

Stroke is a cerebrovascular disease that affects bloodvessels supplying blood to the brain. It occurs when a blood vessel bringing oxygen and nutrients to the brain bursts or is clogged by local thrombus formation or by aggregates of bacteria and fibrin from other sources such as the heart. The inflamed periodontium releases inflammatory cytokines,

LPS, and bacteria into the systemic circulation, and they may promote atherosclerosis and affect blood coagulation, the function of platelets, and PG synthesis, thereby contributing to the onset of stroke. ${ }^{17}$

\section{Infective Endocarditis}

Infective endocarditis is a bacterial infection of the heart valves or the endothelium of the heart. It occurs when bacteria in the bloodstream lodge on abnormal heart valves or damaged heart tissue. Endocarditis occurs rarely in people with normal hearts. However, people who have certain preexisting heart defects are at risk for developing endocarditis when a bacteremia occurs. Infective endocarditis is a serious and often fatal 
systemic disease that has been associated with dental diseases and treatment. Dental procedures, especially extractions and possibly scaling, can be the causation of endocarditis.$^{18}$

\section{Pneumonia}

Pneumonia is an infection of pulmonary parenchyma caused by a wide variety of infectious agents, including bacteria, fungi, parasites, and viruses. Pneumonia can be a life-threatening infection, especially in the old and immunocompromised patient, and is a significant cause of morbidity and mortality in patients of all ages. Bacterial pneumonia results from aspiration of oropharyngeal flora into the lower respiratory tract, failure of host defense mechanisms to eliminate them, multiplication of the microorganisms, and subsequent tissue destruction. It is likely that most pathogens first colonize the surfaces of the oral cavity or pharyngeal mucosa before aspiration. These pathogens can colonize from an exogenous source or emerge following overgrowth of the normal oral flora after antibiotic treatment .Pneumonia can result from infection by anaerobic bacteria. Dental plaque would seem to be a logical source of these bacteria, especially in patients with periodontal disease. ${ }^{19}$ Such patients harbor a large number of subgingival bacteria, particularly anaerobic species. Among the oral bacterial species implicated in pneumonia are A. actinomycetemcomitans , Actinomyces israelii, Capnocytophaga spp. , Eikenella corrodens, Prevotella intermedia, and Streptococcus constellatus . ${ }^{20}$

\section{Low Birth Weight}

Low birth weight, defined as a birth weight of $<2,500 \mathrm{~g}$, is a major public health problem in both developed and developing countries. Pregnancy can influence gingival health. Changes in hormone levels during pregnancy promote an inflammation termed pregnancy gingivitis ${ }^{23}$ This type of gingivitis may occur without changes in plaque levels. ${ }^{24}$ Oral contraceptives may also produce changes in gingival health. Some birth control pill users have a high gingival inflammation level but a low plaque level. Birth control pills may cause changes such as alteration of the microvasculature, gingival permeability, and increased synthesis of estrogen Prostaglandins.$^{22}$ Oral infections also seem to increase the risk for or contribute to low birth weight in newborns. These oral infections induces the inflammatory response with cytokine production which in tern increases the prostaglandins production. ${ }^{21}$ These increased prostaglandins production can leads to pre term labr and low birth weight periodontitis may be a marker for preterm delivery susceptibility as well as a potential risk factor.

\section{Diabetes Mellitus}

Diabetes mellitus is a clinical syndrome characterized by hyperglycemia due to an absolute or relative deficiency of insulin. It affects more than 12 million people in the United States. Diabetes mellitus is characterized by metabolic abnormalities and long-term complications involving the eyes, kidneys, nervous system, vasculature, and periodontium Diabetes is commonly categorized as type 1 , or insulin dependent, and type 2, non-insulin dependent. The fundamental derangement in insulin-dependent diabetes is the hypoproduction of insulin due to destruction of the beta cells of the pancreas. In non-insulin-dependent diabetes, the derangement involves resistance of target tissue to insulin action ${ }^{25}$ glucose-mediated AGE accumulation affects the migration and phagocytic activity of mononuclear and polymorphonuclear phagocytic cells, resulting in the establishment of a more pathogenic subgingival flora. The maturation and gradual transformation of the subgingival microflora into an essentially gram-negative flora will in turn constitute, via the ulcerated pocket epithelium, a chronic source of systemic challenge. This in turn triggers both an "infection-mediated" pathway of cytokine upregulation, especially with secretion of TNF-a and IL-1, and a state of insulin resistance, affecting glucose-utilizing pathways. The interaction of mononuclear phagocytes with AGE-modified proteins induces upregulation of cytokine expression and induction of oxidative stress. Thus, monocytes in diabetic individuals may be "primed" by AGE-protein binding. Periodontal infection challenge to these primed phagocytic cells may, in turn, amplify the magnitude of the macrophage response to AGE-protein, enhancing cytokine production and oxidative stress. ${ }^{26,27}$ Simultaneously, periodontal infection may induce a chronic state of insulin resistance, contributing to the cycle of hyperglycemia, nonenzymatic irreversible glycation, and AGE-protein binding and accumulation, amplifying the classical pathway of diabetic connective tissue degradation, destruction, and proliferation. Hence, the relationship between diabetes mellitus and periodontal disease or infection becomes two way. ${ }^{28}$

\section{Conclusion}

Oral infection, especially periodontitis, are a potential contributing factor to a variety of clinically important systemic diseases. It appears that dental procedures and oral infection meet currently accepted epidemiological criteria for causation of endocarditis, and increases the risk of diseases like pneumoni, endocarditis, diabetes mellitus, low birth weight, etc . A relationship existed between oral health and general 
health .Hence a good oral health is necessary not only to prevent oral disease but also to provide good oral health.

\section{References}

[1] Asikainen, S., and S. Alaluusua. 1993. Bacteriology of dental infections. Eur. Heart J. 14:43-50.

[2] Debelian GJ, Olsen I, Tronstad L. Systemic diseases caused by oral microorganisms. Endod Dent Traumatol 1994; 10: 57-65. (C) Munksgaard, 1994.

[3] Haffajee, A. D., and S. S. Socransky. 1994. Microbial etiological agents of Destructive periodontal diseases. Periodontol. 2000 5:78-111.

[4] Kilian, M. 1982. Systemic disease: manifestations of oral bacteria, p. 832-838. In J. R. McGhee, S. M. Michalek, and G. H. Cassell (ed.), Dental microbiology. Harpers \& Row, Philadelphia, Pa.

[5] Loesche, W. J. 1994. Ecology of the oral flora, p. 307-319. In R. J. Nisengard and M. G. Newman (ed.), Oral microbiology and immunology, 2nd ed. W. B. Saunders, Philadelphia, Pa.

[6] Loesche, W. J., and D. E. Lopatin. 1998. Interactions between periodontal disease, medical diseases and immunity in the older individual. Periodontol. 2000 16:80-105.

[7] Loesche, W. J. 1994. Periodontal disease as a risk factor for heart disease. Compendium 15:976, 978-982, 985-986 passim.

[8] Loesche, W. J. 1997. Association of the oral flora with important medical diseases. Curr. Opin. Periodontol. 4:21-28.

[9] Thoden van Velzen, S. K., L. Abraham-Inpijn, and W. R. Moorer. 1984. Plaque and systemic disease: a reappraisal of the focal infection concept. J. Clin. Periodontol. 11:209-220.

[10] Darveau, R. P., A. Tanner, and R. C. Page. 1997. The microbial challenge in periodontitis. Periodontol. 2000 14:12-32.

[11] McGhee, J. R. 1982. Microbial pathogenic mechanisms, p. 374-387. In J. R. McGhee, S. M. Michalek, and G. H. Cassell (ed.), Dental microbiology. Harper \& Row, Philadelphia, Pa.

[12] Gibbons, R. J., D. I. Hay, W. C. Childs, 3d, and G. Davis. 1990. Role of cryptic receptors (cryptitopes) in bacterial adhesion to oral surfaces. Arch. Oral Biol. 35:107S-114S.

[13] Haffajee, A. D., and S. S. Socransky. 1994. Microbial etiological agents of destructive periodontal diseases. Periodontol. 2000 5:78-111.

[14] Hammond, B. F. 1992. Major bacterial diseases, p. 165-190. In J. Slots and M. A. Taubman (ed.), Contemporary oral microbiology and immunology. Mosby, St. Louis, Mo

[15] Genco, R. J. 1998. Periodontal disease and risk for myocardial infarction and cardiovascular disease. Cardiovasc. Rev. Rep. 19:3437.

[16] Joshipura, K. J., E. B. Rimm, C. W. Douglass, D. Trichopoulos, A. Ascherio, and W. C. Willett. 1996. Poor oral health and coronary heart disease. J. Dent. Res. 75:1631-1636.

[17] Grau, A. J., F. Buggle, C. Ziegler, W. Schwarz, J. Meuser, A. J. Tasman, A. Buhler, C. Benesch, H. Becher, and W. Hacke. 1997. Association between acute cerebrovascular ischemia and chronic and recurrent infection. Stroke 28:1724-1729.

[18] Boon, N. A., and K. A. A. Fox. 1995. Disease of the cardiovascular system, p. 191-312. In C. R. W. Edwards, I. A. D. Bouchier, C. Haslett, and E. R. Chilvers (ed.), Davidson's principles and practice of medicine, 17th ed. Churchill Livingstone, New York, N.Y.

[19] Scannapieco, F. A., and J. M. Mylotte. 1996. Relationships between periodontal disease and bacterial pneumonia. J. Periodontol. 67:1114-1122.

[20] Donowitz, G. R., and G. L. Mandell. 1990. Acute pneumonia, p. 540-555. In G. L. Mandell, R. G. Douglas, and J. E. Bennett (ed.), Principles and practice of infectious diseases. Churchill Livingstone, New York, N.Y.

[21] McCormick, M. C. 1985. The contribution of low birth weight to infant mortality and childhood morbidity. N. Engl. J. Med. 312:82-90.

[22] Hillier, S. L., R. P. Nugent, D. A. Eschenbach, M. A. Krohn, R. S. Gibbs, D. H. Martin, M. F. Cotch, R. Edelman, J. G. Pastorek, 2nd, and A. V. Rao. 1995. Association between bacterial vaginosis and preterm delivery of a low-birth-weight infant. The Vaginal Infections and Prematurity Study Group. N. Engl. J. Med. 333:1737-1742.

[23] Kalkwarf, K. L. 1978. Effect of oral contraceptive therapy on gingival inflammation in humans. J. Periodontol. 49:560-563.

[24] Dasanayake, A. P. 1998. Poor periodontal health of the pregnant woman asa risk factor for low birth weight. Ann. Periodontol. 3:206-212.

[25] Foster, D. W. 1994. Diabetes mellitus, p. 1979-2000. In K. J. Isselbacher, E. Braunwald, J. D. Wilson, J. B. Martin, A. S. Fauci, and D. L. Kasper (ed.), Harrison's principles of internal medicine. McGraw-Hill, New York, N.Y.

[26] Genco, R. J., I. Glurich, V. Haraszthy, J. Zambon, and E. DeNardin. 1998. Overview of risk factors for periodontal disease and implications for diabetes and cardiovascular disease. Compendium 19:40-45.

[27] Grossi, S. G., and R. J. Genco. 1998. Periodontal disease and diabetes mellitus: a two-way relationship. Ann. Periodontol. 3:51-61.

[28] Hugoson, A., H. Thorstensson, H. Falk, and J. Kuylenstierna. 1989. Periodontal conditions in insulin-dependent diabetics. J. Clin. Periodontol. 16: 215-223. 